\title{
ÉTUDE DES COUCHES MINCES ET DES SURFACES PAR RÉFLEXION RASANTE, SPÉCULAIRE OU DIFFUSE, DE RAYONS $X$
}

\author{
P. CROCE et L. NÉVOT
}

Institut d'Optique, Laboratoire associé au C. N. R. S., Université de Paris-Sud, Bât. 503, 91405 Orsay, France

\begin{abstract}
Résumé. - Nous savons que les équations de Fresnel appliquées à des dioptres supposés, parfaitement plans et homogènes, ne permettent pas de rendre compte des courbes de réflexion spéculaire fournies par des échantillons massifs ou en couche mince. Nos considérations sur divers processus de diffusion élastique ou inélastique, montrent que seule la diffusion élastique introduite par les irrégularités ou inhomogénéités de surface est importante pour l'analyse de ces courbes. Nous proposons quelques modèles de rugosité et en précisons les domaines de validité aussi bien pour la réflexion spéculaire, que pour la réflexion diffuse (suivant la loi des réseaux). Ces modèles sont comparés avec les courbes expérimentales obtenues sur des échantillons massifs de titane, de silicium et de germanium, avec les rayonnements $\mathrm{K} \alpha_{1}$ du cuivre ou du chrome.
\end{abstract}

\begin{abstract}
The Fresnel formulae applied to optical surfaces, assumed to be perfectly plane and homogeneous, do not correctly explain the specular reflection curves obtained with bulk specimens or thin films. Considerations on various scattering processes (either elastic or inelastic) show that only elastic scattering, due to surface roughness or structural inhomogeneity, must play a role in the analysis of the reflection curves.

In this paper some roughness models are presented and their validity range is given for both specular and diffuse reflection. Theoretical curves derived from these models are compared with experiments carried out on bulk specimen of titanium, silicon and germanium using the copper or chromium $K \alpha_{1}$ radiations.
\end{abstract}

1. Introduction. - Les rayonnements électromagnétiques sont utilisés depuis très longtemps pour l'étude des matériaux, et ce pour de multiples raisons. Citons, en particulier, la possibilité de produire assez facilement des faisceaux suffisamment intenses, et d'obtenir, dans de nombreux cas, un bon rapport diffusion élastique-diffusion inélastique : ceci permet, entre autres, d'observer des interférences et de déterminer, par suite, les caractéristiques géométriques des échantillons.

Lorsqu'on s'intéresse plus particulièrement à la surface des matériaux, on se trouve souvent en présence de couches superficielles d'épaisseurs comprises entre quelques dizaines et quelques centaines d'angströms, c'est-à-dire faibles devant les longueurs d'onde du visible. Ceci incite à utiliser des rayonnements de longueurs d'onde beaucoup plus courtes. On pourrait se placer dans le domaine allant du proche $\mathrm{U}$. V. jusqu'aux rayons $\mathrm{X}$, mais l'atténuation due à l'absorption par l'air devient trop forte; il est alors indispensable de travailler sous vide, d'où une certaine complication expérimentale, qui limite en fait leur exploitation. On est donc amené au domaine des rayons $\mathrm{X}$, domaine pour lequel les indices de réfraction des matériaux sont très voisins de 1 .

Pour obtenir des faisceaux réfléchis d'intensité mesurable, il est nécessaire d'attaquer les surfaces sous angles rasants, et la méthode d'analyse des surfaces par réflexion rasante, spéculaire ou diffuse, de rayons $\mathrm{X}$, consiste ainsi à enregistrer et à analyser les variations de l'intensité du faisceau réfléchi en fonction de l'angle d'incidence sur la surface. Le fait de travailler à angle variable représente un avantage indéniable par rapport à d'autres méthodes d'analyse de surface, car on peut dire, du moins dans le cas de la réflexion spéculaire, que cela revient à faire varier le couplage avec la matière.

Nous nous proposons ici de passer en revue quelques-uns des résultats qu'il est possible d'atteindre par cette méthode de réflexion rasante de rayons $X$ et d'essayer de justifier les techniques adoptées pour l'analyse des courbes expérimentales.

2. Généralités et dispositif expérimental.-2.1 GÉNÉRALITÉs. - Dans le domaine de fréquence des rayons $X$ moyens, l'indice de réfraction d'un matériau de numéro et masse atomiques, $Z$ et $A$ respectivement, de masse spécifique $\rho$, pour un rayonnement de longueur d'onde $\lambda$, est donné par la relation

$$
n=1-\delta-i \beta
$$

avec

$$
\delta=2,7019 \times 10^{10} \frac{\rho \lambda^{2}}{A} f_{0} \text { (unités C. G. S.) }
$$


où $f_{0}$ représente le facteur de diffusion atomique. Dans le cas de la diffusion centrale, $f_{0}$ s'écrit sous la forme

$$
f_{0}=Z+\Delta f_{0}^{\prime},
$$

si $\Delta f_{0}^{\prime}$ désigne la partie réelle du terme de correction de dispersion anomale. De nombreuses études théoriques ont été consacrées à la détermination de ces termes correctifs pour les longueurs d'ondes les plus usuelles. Nous citerons en particulier celles de Dauben et Templeton [1], de Cromer [2] et de Cromer et Liberman [3].

En ce qui concerne $\beta$, on le déduit directement, en général, de la mesure du coefficient d'absorption linéaire $\mu \mathrm{du}$ matériau pris à l'état massif, par la relation

$$
\beta=\frac{\lambda}{4 \pi} \mu
$$

Pour le rayonnement $\mathrm{K}_{\alpha_{1}}$, du cuivre, $\delta$ est de l'ordre de quelque $10^{-5}$ et $\beta$ de quelque $10^{-6}$. Ainsi, l'angle limite de réflexion totale (elle serait effectivement totale, s'il n'y avait pas d'absorption) n'excède pas quelques dizaines de minutes d'arc. Pour obtenir des faisceaux réfléchis mesurables il est donc nécessaire de travailler sous incidence quasi rasante, donc de disposer d'un goniomètre très précis.

2.2 DisPositif EXPÉRIMENTAL. - Un schéma synoptique de l'ensemble expérimental est donné ci-dessous. Le tube à rayons $X$ est à foyer optique linéaire. Son refroidissement est assuré par l'intermédiaire d'un circuit fermé, alimenté en eau régulée à la fois en

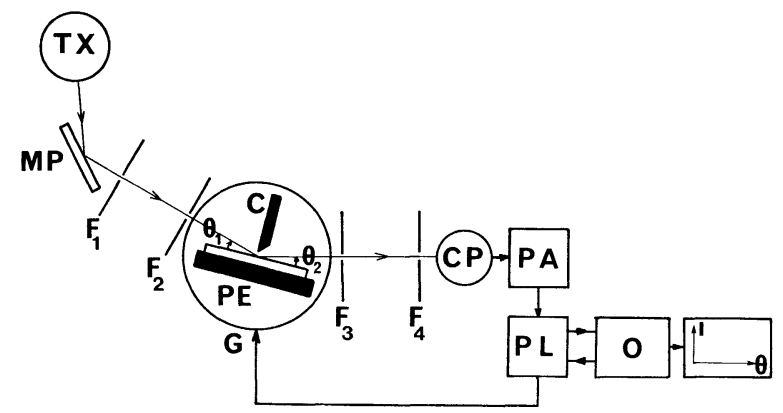

SCHÉMA 2.2. - Dispositif expérimental. T. X. : Tube à rayons X ; M. P. : Monochromateur plan de quartz $\left(\alpha=0^{\circ}\right)$; G. : Goniomètre ; C. : Couteau escamotable pour effectuer les réglages ; P. E. : Platine porte-échantillon (l'échantillon y est maintenu par aspiration) ; C. P. : Compteur proportionnel au xénon ; P. A. : Préamplificateur ; P. L. : Organe périphérique de liaison ; O. : Ordinateur.

température et en débit. Le rayonnement issu de l'anticathode de cuivre (notons que pour quelques expériences nous avons utilisé aussi le chrome) tombe sur un monochromateur plan de quartz $\left(\alpha=0^{\circ}\right)$ orienté de telle sorte que le doublet $\mathrm{K}_{\alpha}$ soit sélectionné. La raie $K_{\alpha_{1}}$ est ensuite isolée à l'aide d'une première fente $F_{1}$, de $60 \mu \mathrm{m}$ de large, située à environ $15 \mathrm{~cm}$ de l'axe du monochromateur.
Comme le dispositif expérimental fonctionne essentiellement en faisceau parallèle on pourrait, afin d'augmenter le nombre de photons reçus, donc de diminuer le temps de mesure, utiliser des sources de rayons $\mathrm{X}$ étendues en conservant la même luminance. Toutefois, à résolution égale, ceci exigerait d'une part un goniomètre encore plus encombrant et d'autre part des échantillons de plus grande surface. Or, les conditions de planéité des surfaces à examiner dans ce type d'étude sont assez sévères. Les difficultés d'obtenir sur de grandes dimensions : soit une bonne planéité, par polissage à partir d'échantillons massifs (à noter aussi, que dans ce cas, le coût de revient du matériau est un facteur non négligeable), soit une bonne homogénéité, aussi bien en épaisseur qu'en densité, pour les couches déposées sur substrats (de verre par exemple), font que l'on doive réaliser un compromis sur les divers paramètres géométriques.

Du fait de l'incidence rasante du faisceau incident, la longueur éclairée sur l'échantillon reste importante pour les incidences faibles, même avec une fente source de $60 \mu \mathrm{m}$ de large. Ainsi, pour l'angle d'incidence qui correspond à l'angle limite de réflexion totale d'un verre poli de type borosilicate Crown $\left(\delta=8,0 \times 10^{-6}\right.$ pour $\lambda=1,5405 \AA$, soit

$$
\left.\theta_{\mathrm{c}}=4 \mathrm{mrd}=825^{\prime \prime}\right)
$$

cette longueur est de $1,5 \mathrm{~cm}$. C'est pourquoi nous avons choisi, pour le montage expérimental, une hauteur utile de fente de $1,5 \mathrm{~cm}$, laquelle représente également une bonne adaptation à la hauteur de la source de rayons $X$.

Les deux fentes $F_{2}$ et $F_{3}$ permettent d'éliminer les diffusions parasites éventuelles et de contrôler la divergence verticale du faisceau.

Les rotations de la platine et du bras porte-compteur sont accomplies par l'intermédiaire de deux moteurs pas à pas. La précision des déplacements obtenus est de $\pm 2,5 \mu \mathrm{m}$. Or, la précision angulaire souhaitée est de $\pm 1^{\prime \prime}$ : en effet, dans le cas des couches minces d'épaisseur de l'ordre de $1500 \AA$ les franges d'interférences sont extrêmement serrées, et d'autre part, dans les mesures précises d'indice pour les matériaux très peu absorbants, la chute d'intensité à la limite de réflexion totale est très brutale, ce qui requiert, pour l'enregistrement de la courbe expérimentale, des pas angulaires très petits (disons, $5^{\prime \prime}$ comme ordre de grandeur).

La longueur requise pour les bras de transmission est donc de $50 \mathrm{~cm}$ environ. Par suite, la fente d'analyse $F_{4}$, de même largeur que la fente $F_{1}$, est placée à $50 \mathrm{~cm}$ de l'axe de la platine. Ainsi la fraction d'angle solide utile pour la détection des photons réfléchis n'est que de $3 \times 10^{-5}$ environ.

L'asservissement par ordinateur des moteurs pas à pas permet de réaliser n'importe quelle combinaison entre $\theta_{1}$, angle d'attaque du faisceau incident, 
et $\theta_{2}$, angle de détection du faisceau réfléchi, ces angles étant définis ici par rapport à la surface moyenne de l'échantillon. Ainsi, dans le cas de la réflexion spéculaire, nous avons constamment $\theta_{1}=\theta_{2}(=\theta)$. Pour la réflexion diffuse, nous pouvons faire varier $\theta_{2}$ en laissant $\theta_{1}$ fixe, ou bien encore, faire évoluer $\theta_{1}$ et $\theta_{2}$ de telle sorte que la loi des réseaux soit respectée c'est-à-dire en gardant constant $\left(\cos \theta_{1}-\cos \theta_{2}\right)$.

Les résultats numériques (valeurs des angles $\theta_{1}$ et $\theta_{2}$, et de l'intensité correspondante exprimée en nombre d'impulsions/s), ainsi que le tracé de la courbe expérimentale, sont visualisés directement sur imprimante.

Selon les valeurs de $\theta_{1}$, l'intensité du faisceau réfléchi varie de façon considérable (de 1 à $10^{7}$ ). Aussi, pour conserver la linéarité de réponse du compteur proportionnel, nous utilisons des écrans absorbants, de façon à ne pas dépasser $10^{3}$ coups $/ \mathrm{s}$. D'un autre côté, pour obtenir une assez bonne précision statistique (au moins, meilleure que $10 \%$ ) sans qu'il soit nécessaire de disposer pour cela de temps de comptage excessifs dans le cas des flux les plus faibles (on rappelle que le bruit de fond n'est que de 5 coups/min environ) le temps imparti à chaque mesure est adapté au taux de photons (nombre de photons/s) qui pénètre dans le compteur à la suite d'un test portant sur un petit nombre d'impulsions.

2.3 ANAlyse D'Une Courbe eXPÉRIMENTAle. - Sur la figure 1 nous reproduisons une courbe caractéristique de réflexion spéculaire, fournie par une couche mince d'or attaquée par le rayonnement $K_{\alpha_{1}} d u$ cuivre $(\lambda=1,5405 \AA)$. Cette couche a été obtenue par évaporation en ultra-vide $\left(10^{-9} \mathrm{~mm}\right.$ de mercure) sur substrat de float-glass (verre coulé sur bain d'étain) brut de fabrication (hormis le nettoyage de surface habituel). La déposition est suivie d'un recuit à $150^{\circ} \mathrm{C}$ pendant une heure environ.

La courbe expérimentale fait apparaître deux zones angulaires bien distinctes. Pour la première, l'intensité ne décroît que lentement lorsque $\theta$ croît, puis chute brutalement au voisinage de $\theta_{\mathrm{c}}^{\mathrm{Au}}$, angle critique de réflexion totale pour l'or. Ceci permet d'estimer $\delta_{\mathrm{Au}}$ puisque $\theta_{\mathrm{c}}$ et $\delta$ sont liés par la relation $\theta_{\mathrm{c}}^{2}=2 \delta$. Cette zone de réflexion presque totale est suivie d'une zone de réflexion vitreuse où apparaissent éventuellement certaines oscillations, ou franges d'interférences, lorsque l'épaisseur de la couche mince est suffisamment petite (la limite supérieure se situe vers $2000 \AA$ ). De telles franges traduisent les battements entre les faisceaux de rayons $\mathrm{X}$ réfléchis par les dioptres respectifs couche-support et couche-air. On remarque que les franges lointaines sont sensiblement équidistantes angulairement. L'épaisseur $e$ de la couche peut être alors déduite de la mesure de cet interfrange $\Delta \theta$ par la relation

$$
e=\frac{\lambda}{2 \Delta \theta}
$$

Pour essayer de simuler complètement la courbe expérimentale, on utilise souvent les équations de Fresnel [4], en supposant, dans une première étape, les dioptres évoqués précédemment, parfaitement plans et homogènes. L'indice de réfraction du substrat ayant été, si possible, déterminé préalablement par simulation de la courbe de réflexion spéculaire du substrat nu, il ne reste plus que deux paramètres; indice de réfraction et épaisseur de la couche, sur lesquels on joue, à partir des valeurs approximatives telles que déduites précédemment, pour parfaire la simulation. Or nous constatons que si les positions angulaires des minimums et maximums sont bien respectées, ce qui permet de définir une épaisseur moyenne très précise, ici $e=(236,5 \pm 1) \AA$, ainsi que la forme de la courbe dans la zone de réflexion totale (hormis la zone de rasance presque parfaite où les effets de géométrie sont pratiquement inévitables), la décroissance de l'intensité expérimentale dans la zone de réflexion vitreuse est nettement plus rapide que ne le prédisent les équations de Fresnel. En outre la valeur de $\delta$, ainsi trouvée, est souvent quelque peu inférieure à la valeur théorique. Dans

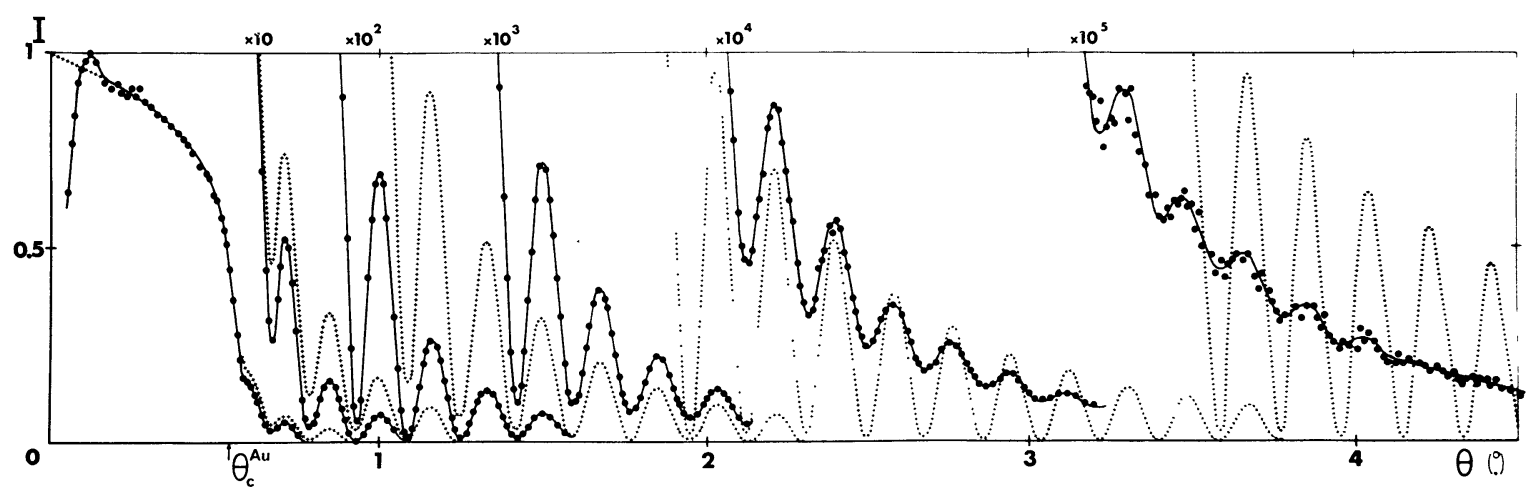

Fig. 1. - Courbe expérimentale de réflexion spéculaire (————- $\longrightarrow$ ) obtenue avec une couche mince d'or déposée en ultra-vide $\left(10^{-9} \mathrm{mmHg}\right)$ sur substrat de float-glass, puis recuite pendant une heure environ à $150^{\circ} \mathrm{C}(\lambda=1,5405 \AA)$. La courbe théorique $(+++++)$ est relative au modèle plan parfait avec comme indices, pour $1^{\prime}$ 'or : $\delta=46 \times 10^{-6}$ et $\beta=4,9 \times 10^{-6}$, et pour le verre : $\delta=7,7 \times 10^{-6}, \beta=1,3 \times 10^{-7}$, et comme épaisseur pour la couche $e=236,5 \AA$. 
notre exemple, nous obtenons $\delta_{\mathrm{Au}}=(46 \pm 1) 10^{-6}$ alors que la valeur théorique compte tenu des corrections de dispersion anomale est de $46,5 \times 10^{-6}$ et la valeur classique de $49,6 \times 10^{-6}$ pour le rayonnement $\mathrm{K}_{\alpha_{1}}$ du cuivre. D'autre part, pour l'indice du float-glass nous avons repris la valeur de 7,7 $\times 10^{-6}$ que nous avons déjà déterminée (L. Névot et P. Croce [5]) ce qui diffère fortement de la valeur calculée à partir de la composition chimique donnée par le fabricant (soit $8,3 \times 10^{-6}$ après corrections de dispersion anomale).

Il est cependant évident que l'hypothèse de dioptres parfaitement plans et homogènes n'est pas très réaliste. Toutefois avant de la mettre en cause, nous essaierons de voir quels effets de perturbation peuvent résulter de certains processus de diffusion quant à l'interprétation de nos courbes expérimentales. Nous examinerons d'abord le cas de la diffusion inélastique, puis la diffusion élastique en volume en y incluant, pour des raisons de similitude, la diffusion thermique, pour terminer par la diffusion engendrée par les irrégularités de surface.

3. Influence des divers processus de diffusion. 3.1 SUR LA DIFFUSION inélastique. - Le rayonnement, qui pénètre dans l'échantillon, est absorbé après la traversée d'une certaine épaisseur. D'où une réémission possible de photons, certains d'entre eux pouvant être reçus par le détecteur. Mais pour des photons incidents dont l'énergie est de quelques $\mathrm{keV}$, l'absorption primaire principale provient de l'effet photoélectrique. La réémission s'effectuera par un électron Auger, ou, et c'est le cas qui nous intéresse, par un photon de fluorescence. L'électronique de mesure, qui suit le compteur proportionnel, comportant un sélecteur d'amplitude, il est nécessaire que l'énergie du photon de fluorescence soit suffisamment voisine de celle des photons incidents pour qu'il puisse être compté. On ne peut donc avoir, au pire, qu'un seul photon de fluorescence par photon incident.

Admettons, par ailleurs, que l'émission de ces photons se fasse, dans l'espace, d'une façon isotrope (on peut concevoir en effet que dans un processus aussi complexe, l'impulsion de ces photons dépende peu des propriétés des particules incidentes). Le flux correspondant serait donc équivalent à l'angle solide de détection, soit $3 \times 10^{-5}$ en valeur relative par rapport aux $4 \pi$ stéradians de la sphère, ce qui le situe sensiblement au niveau du bruit de fond (on notera cependant que nos hypothèses ont été particulièrement généreuses pour la diffusion inélastique).

Considérons maintenant le cas de la diffusion Compton du photon incident. Nous savons que dans le cas de photons incidents de faible énergie, un tel processus n'est pas extrêmement directif. Pour fixer un ordre de grandeur, nous rappelons que pour un électron libre, la diffusion vers l'avant n'est qu'une fois et demie plus intense que la diffusion moyenne dans tout l'espace. Comme en outre la diffusion sous faibles angles devient très faible lorsque les électrons sont liés, nous pouvons conclure, en considérant que la diffusion inélastique n'introduit qu'une sorte de bruit de fond supplémentaire, puisque très faible et variant peu avec l'angle $\left(\theta_{1}+\theta_{2}\right)$.

3.2 SUR LA DIFFUSION ÉLASTIQUE EN VOLUME ET LA DIFFUSION THERMIQUE. - Nous examinons maintenant le cas de la diffusion élastique engendrée par le volume irradié dans l'échantillon, en supposant ce volume homogène. En outre, comme la contribution de ce processus à l'intensité réfléchie ne risque de devenir non négligeable que lorsque les angles d'incidence prennent des valeurs élevées, nous admettons que le rayonnement pénètre dans la matière sans réfraction. Appelons $E_{0}$ l'amplitude du champ électrique de l'onde plane incidente, de vecteur d'onde dans le vide $\mathbf{k}_{\mathbf{i}}\left(\right.$ avec $\left.\left|\mathbf{k}_{\mathbf{i}}\right|=k_{0}\right)$. Nous prenons un trièdre de référence (voir schéma) tel que le plan $x O y$ soit

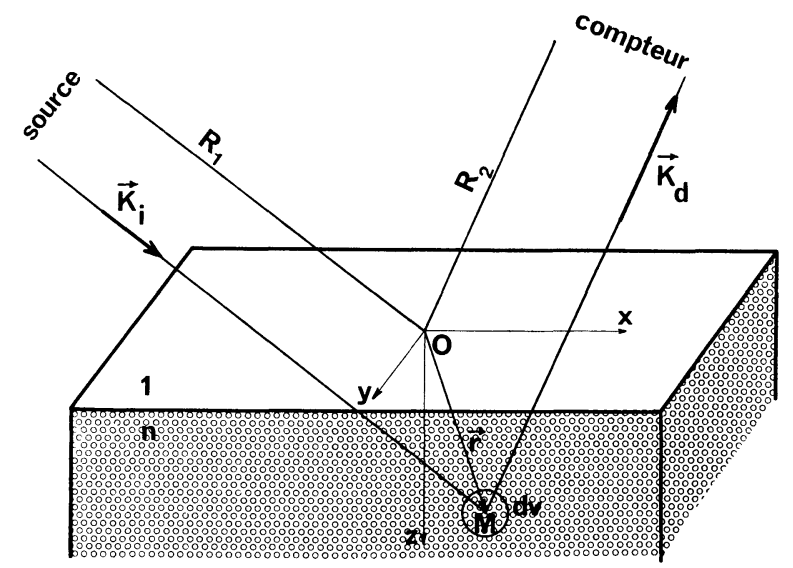

SCHÉMA 3.2.

confondu avec la surface moyenne de l'échantillon, l'origine $O$ se trouvant à la distance $R_{1}$ de la fente source $\mathrm{F}_{1}$ et l'axe $O z$ étant orienté positivement vers la matière. Au point $M$, défini par le vecteur $\mathbf{r}$, l'amplitude du champ électrique de l'onde incidente peut s'écrire sous la forme :

$$
\mathbf{E}_{\boldsymbol{i}}=\tau_{\mathbf{i}} \mathbf{E}_{0} \exp \left(-2 \pi i \mathbf{k}_{\mathbf{i}} \cdot \mathbf{R}_{1}\right) \exp \left(-2 \pi i \mathbf{k}_{\mathbf{i}}^{\prime} \cdot \mathbf{r}\right)
$$

où $\tau_{\mathbf{i}}$ représente le facteur de transmission en amplitude pour le champ électrique, au niveau de l'interface et pour l'incidence $\theta_{\mathbf{i}}$, et $\mathbf{k}_{\mathbf{i}}^{\prime}$ le vecteur d'onde de l'onde incidente dans le milieu d'indice $n(n=1-\delta-i \beta)$. $\mathbf{k}_{\mathbf{i}}^{\prime}$ et $\mathbf{k}_{\mathbf{i}}$ sont liés par les relations :

$$
k_{\mathbf{i}}^{\prime 2}=n^{2} k_{\mathbf{i}}^{2} \quad \text { et } \quad\left(\mathbf{k}_{\mathbf{i}}^{\prime}\right)_{\mathbf{t}}=\left(\mathbf{k}_{\mathbf{i}}\right)_{\mathbf{t}}
$$

cette dernière traduisant l'égalité des composantes tangentielles (c'est-à-dire des projections dans le plan $x O y$ ). On en déduit la relation sur les composantes normales,

$$
\left(k_{\mathrm{i}}^{\prime}\right)_{\mathrm{t}}^{2}=\left(k_{\mathrm{i}}\right)_{\mathrm{z}}^{2}\left(1-\frac{\theta_{\mathrm{c}}^{2}}{\theta^{2}}-2 i \beta \frac{k_{0}^{2}}{k_{\mathrm{iz}}^{2}}\right) .
$$


Pour les incidences suffisamment élevées, $\theta_{\mathrm{c}}^{2} / \theta^{2}$ est négligeable devant 1 et comme, d'autre part, $\beta$ n'excède pas quelque $10^{-6}$, on peut écrire :

$$
k_{\mathrm{i} z}^{\prime} \cong k_{\mathrm{i} z}-i \beta \frac{k_{0}^{2}}{k_{\mathrm{i} z}} .
$$

L'expression (1) devient alors :

$\mathbf{E}_{\mathbf{i}}=\tau_{\mathbf{i}} \mathbf{E}_{0} \exp \left[-2 \pi i \mathbf{k}_{\mathbf{i}} \cdot\left(\mathbf{R}_{1}+\mathbf{r}\right)-2 \pi \beta \frac{k_{0}^{2}}{k_{\mathbf{i} z}} z\right]$.

L'expression du champ de l'onde diffusée (de vecteur $\mathbf{k}_{\mathrm{d}}$ ) dans la fente du compteur (située à la distance $R_{2}$ de $O$ ) et engendrée par le volume $\mathrm{d} v$ où règne une fluctuation d'indice $\Delta\left(n^{2}\right)$ est alors, d'après le principe de réciprocité (voir P. Croce, L. Névot, B. Pardo [6]) :

$$
\begin{aligned}
\mathrm{d} \mathbf{E}_{\mathrm{d}}=-4 & \pi^{2} \frac{k_{0}^{2}}{R_{2}} \frac{\Delta\left(n^{2}\right)}{4 \pi} \mathrm{d} v \tau_{\mathrm{i}} \tau_{\mathrm{d}} \mathbf{E}_{\mathrm{i}} \times \\
& \times \exp \left[-2 \pi i \mathbf{k}_{\mathrm{d}} \cdot\left(\mathbf{R}_{2}-\mathbf{r}\right)+2 \pi \beta \frac{k_{0}^{2}}{k_{\mathrm{d} z}} z\right]
\end{aligned}
$$

soit compte tenu de (2) :

$\mathrm{d} \mathbf{E}_{\mathrm{d}}=c \tau_{\mathrm{i}} \tau_{\mathrm{d}} \mathbf{E}_{0} \exp \left[-2 \pi i\left(\mathbf{k}_{\mathrm{i}}-\mathbf{k}_{\mathrm{d}}\right) \cdot \mathbf{r}\right] \exp (-a z)$

avec :

$\left\{\begin{array}{l}a=2 \pi \beta k_{0}^{2}\left(\frac{1}{k_{\mathrm{i} z}}-\frac{1}{k_{\mathrm{d} z}}\right)=2 \pi \beta k_{0}\left(\frac{1}{\sin \theta_{\mathrm{i}}}+\frac{1}{\sin \theta_{\mathrm{d}}}\right) \\ c=-\frac{4 \pi^{2} k_{0}^{2}}{R_{2}} \cdot \frac{\Delta\left(n^{2}\right)}{4 \pi} \mathrm{d} v \exp \left[-2 \pi i\left(\mathbf{k}_{\mathrm{i}} \cdot \mathbf{R}_{1}+\mathbf{k}_{\mathrm{d}} \cdot \mathbf{R}_{2}\right)\right] .\end{array}\right.$

L'expression (3) montre que tout se passe comme s'il n'y avait aucune absorption et que la zone diffusante était éclairée uniformément avec un champ excitateur d'amplitude $\tau_{\mathrm{i}} \tau_{\mathrm{d}} \mathrm{E}_{0}$, sur une profondeur 3 égale à $1 / 2 a$ (le facteur $\frac{1}{2}$ est imposé par les considérations sur les intensités et non sur les amplitudes diffusées). Signalons que cette profondeur utile 3 est égale, dans le cas de la réflexion spéculaire, à la moitié de la profondeur de pénétration définie par A. T. Alichanow et L. A. Arimowic [7] ou L. G. Parratt [8] comme étant équivalente à la cote $z(1 / e)$ pour laquelle l'intensité du rayonnement réfracté incident est réduite par rapport à sa valeur au niveau de l'interface par le facteur $1 / e$.

Nous indiquons, tableau I, les valeurs de 3 (exprimées en microns) relatives à quelques matériaux éclairés par le rayonnement $K_{\alpha_{1}}$ du cuivre (pour le titane, la seconde ligne concerne le rayonnement $\mathbf{K}_{\alpha_{1}}$ du chrome) sous incidence rasante ou sous incidence normale, dans le cas de la réflexion spéculaire. Les valeurs précédées d'un astérisque doivent être considérées comme approximatives car $\theta$ est alors assez proche de $\theta_{\mathrm{c}}$.

Nous noterons que les valeurs de 3 indiquées dans la colonne de droite du tableau I correspondent aussi à la moitié des épaisseurs optimales à utiliser pour les matériaux considérés dans le cas de la diffusion centrale par transmission. Remarquons aussi que le volume éclairé utile, pour la réflexion spéculaire sous incidence élevée $\left(\theta \gg \theta_{c}\right)$, reste constant : en effet, la surface $S$ éclairée sur l'échantillon varie comme $1 / \sin \theta$ et l'épaisseur comme $\sin \theta$. Par conséquent la diffusion élastique en volume se comporte, là encore, comme un bruit de fond, si ce volume est homogène.

Vérifions que l'on obtient le même résultat dans le cas de la diffusion thermique, où les fluctuations de constante diélectrique $\Delta \varepsilon$, donc d'indice, sont dynamiques au lieu d'être statiques comme précédemment.

Les vecteurs d'onde $\mathbf{K}$ des oscillations thermiques qui vont contribuer à la diffusion sont voisins de $\mathbf{k}_{\mathbf{i}}-\mathbf{k}_{\mathrm{d}}(=\mathbf{k})$. Nous prendrons un volume de quantification parallélépipédique pour ces oscillations, de telle manière que ce volume coïncide en surface avec la surface éclairée (et vue par le récepteur) que nous supposerons de forme rectangulaire $(S=X . Y)$ telle que les conditions de Born von Karman

$$
k_{x} \cdot X=2 \pi p, \quad k_{y} \cdot Y=2 \pi q
$$

(avec $p$ et $q$ entiers) soient satisfaites, et de profondeur $Z$ grande devant la profondeur éclairée. L'amplitude

\begin{tabular}{|c|c|c|c|c|c|}
\hline Substance & $10^{6} \beta$ & 10 & $2^{0}$ & $4^{0}$ & $90^{\circ}$ \\
\hline Verre & 0,13 & 0,822 & 1,64 & 3,29 & 47,15 \\
\hline Silicium & 0,173 & 0,618 & 1,236 & 2,47 & 35,43 \\
\hline Germanium & 0,496 & $* 0,215$ & 0,431 & 0,869 & 12,35 \\
\hline Titane & $\begin{array}{l}1,136 \\
4,635\end{array}$ & $\begin{array}{l}* 9,41 \times 10^{-2} \\
* 3,43 \times 10^{-2}\end{array}$ & $\begin{array}{l}0,188 \\
6,85 \times 10^{-2}\end{array}$ & $\begin{array}{l}0,376 \\
0,137\end{array}$ & $\begin{array}{l}5,39 \\
1,96\end{array}$ \\
\hline Or & 4,92 & $* 2,17 \times 10^{-2}$ & $4,34 \times 10^{-2}$ & $8,69 \times 10^{-2}$ & 1,24 \\
\hline
\end{tabular}
de chaque onde est proportionnelle à $1 / \sqrt{S Z}$. Il faudra que la composante tangentielle de $\mathbf{K}$ coïncide

TABLEAU I 


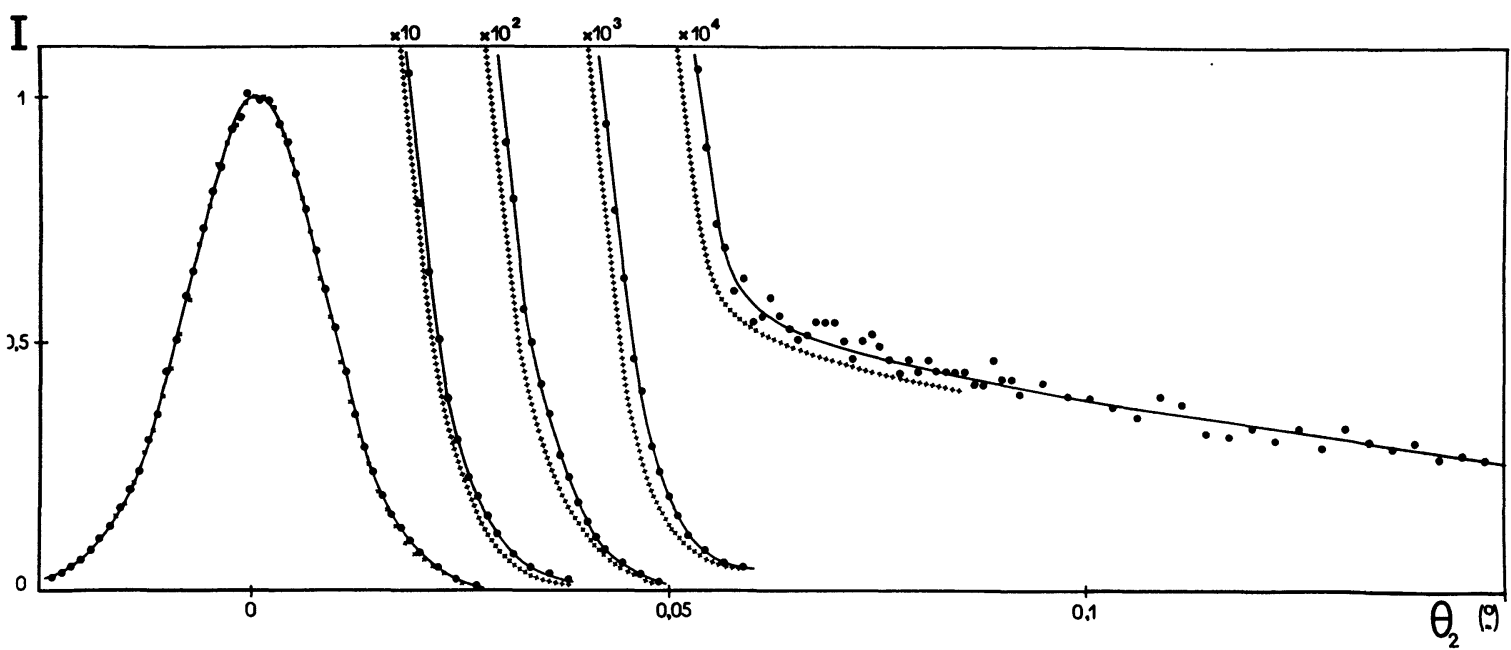

Fig. 2. - Comparaison entre l'intensité du faisceau direct $(+++++)$ et celle transmise à travers une lamelle de float-glass (d'épaisseur $0,16 \mathrm{~mm}$ ) sous incidence normale (- $\bullet \bullet-$ ), après compensation d'absorption.

avec celle de $\mathbf{k}$ et la contribution de chacune des oscillations thermiques sera proportionnelle à :

$$
\begin{aligned}
\frac{1}{S Z} \mid \int_{0}^{Z} \exp \left(2 \pi i K_{z} z\right) \exp (- & \left.2 \pi i k_{z} z-a z\right)\left.\mathrm{d} z\right|^{2}= \\
= & \frac{S}{Z} \frac{1}{4 \pi^{2}\left(K_{z}-k_{z}\right)^{2}+a^{2}}
\end{aligned}
$$

en supposant les propriétés élastiques indépendantes de $z$ et $k_{z} \gg a$.

Les composantes $K_{z}$ étant séparées, dans l'espace réciproque, de $1 / Z$, la contribution de l'ensemble des ondes sera donnée par:

$$
S \int_{-\infty}^{+\infty} \frac{\mathrm{d} K_{z}}{4 \pi^{2}\left(K_{z}-k_{z}\right)^{2}+a^{2}}=\frac{S}{2 a}=S 3 .
$$

On retrouve ainsi, ce qui est naturel, le même volume équivalent que plus haut et on ne séparera plus ces deux diffusions.

3.3 DiFfuSION PAR LES IRRÉGULARITÉS DE SURFACE. - Du fait de la géométrie particulière du montage expérimental dans nos études par réflexion rasante, les effets de surface sont largement favorisés. Aussi pour une incidence rasante de $2^{\circ}$ sur un substrat de verre, la surface éclairée représente $25,78 \mathrm{~mm}^{2}$ alors que la profondeur de pénétration 3 vaut $1,64 \mu \mathrm{m}$. Par contre, dans une étude de diffusion centrale par transmission sur ce même verre, la surface éclairée n'est plus que de $0,9 \mathrm{~mm}^{2}$, et la longueur éclairée $(60 \mu \mathrm{m})$ devient comparable à $3_{\text {opt }}(94,30 \mu \mathrm{m})$.

Sur la figure 2 nous comparons l'intensité du faisceau transmis à travers une lamelle de float-glass (polie sur les deux faces), d'épaisseur égale à $0,16 \mathrm{~mm}$, et disposée perpendiculairement au faisceau, à celle du faisceau direct $\left({ }^{1}\right)$ (une fois la lamelle retirée), ceci après avoir

(1) Il faudrait d'ailleurs faire la convolution de cette courbe avec les courbes théoriques de réflexion spéculaire avant de comparer ces dernières aux courbes expérimentales. effectué la correction due à l'absorption dans le verre. (Comme les trajets parcourus au total par les rayonnements diffusés sont pratiquement équivalents à l'épaisseur de la lame, ils subissent sensiblement la même absorption; il suffit alors de ramener l'intensité du pic central à celle du faisceau direct.) On peut ainsi constater que les deux courbes sont extrêmement voisines et que, par suite, la diffusion en volume est négligeable devant celle, elle-même très faible, engendrée par l'air et, surtout, par les fentes. Précisons en outre, que si l'on voulait déduire l'intensité diffusée en volume dans les expériences de réflexion spéculaire rasante, de celle mesurée en diffusion centrale, il faudrait comparer les volumes diffusants. Avec les valeurs données précédemment, il est aisé de montrer que cette intensité devrait être divisée par 3,4. Nous pouvons donc affirmer que les expériences de réflexion rasante, spéculaire ou diffuse, de rayons $X$, sont surtout sensibles aux effets de surface, dans la mesure évidemment où les dépôts étudiés sont homogènes, ou bien, présentent une répartition de densité électronique suffisamment stratifiée (dans le cas d'empilement de couches minces, par exemple). Cette dernière hypothèse revient à considérer l'échantillon comme constitué de un ou plusieurs dioptres parfaitement homogènes. Admettons en outre qu'ils soient parfaitement plans. Nous avons déjà vu que, dans ce cas, les équations de Fresnel ne permettent pas de simuler correctement les courbes expérimentales de réflexion spéculaire. Le désaccord observé quant aux valeurs d'indice et à la décroissance de l'intensité moyenne du faisceau réfléchi dans l'exemple de la couche mince d'or déposée sur float-glass (Fig. 1) se retrouve aussi pour l'échantillon de titane, analysé soit avec le rayonnement $\mathrm{K}_{\alpha_{1}}$ du cuivre (Fig. $3 a$ ) soit avec celui du chrome (Fig. $3 b$ ). Nous obtenons, en effet, pour les valeurs de $\delta$, relatives à ces deux longueurs d'onde $(12,8 \pm 0,2) \times 10^{-6}$ et $(25,8 \pm 0,4) \times 10^{-6}$, respectivement, alors que les valeurs théoriques clas- 


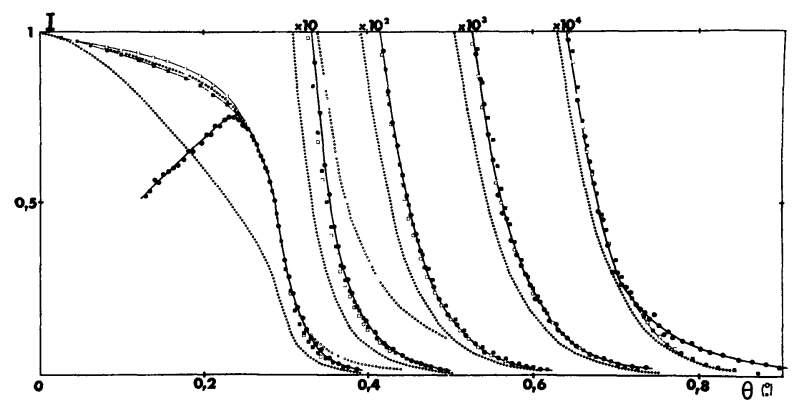

Fig. 3a. - Courbe expérimentale de réflexion spéculaire (-๑-—--) obtenue avec le rayonnement $\mathrm{CuK}_{\alpha_{1}}$ sur un échantillon massif de titane. On présente également les courbes théoriques de simulation données par les modèles suivants : i) modèle plan parfait $(0000000)$ avec pour indice du titane $\delta=12,8 \times 10^{-6}, \quad \beta=1,14 \times 10^{-6}$; ii) modèle C. P. S. (口سD) où l'indice varie suivant la fonction d'erreur, avec $L=96 \AA$ (soit $\sqrt{\left\langle z^{2}\right\rangle}=21,2 \AA$ ); iii) modèle déduit de l'expression théorique (5) $(\square \square \square \square)$ avec $\sqrt{\left\langle z^{2}\right\rangle}=21,9 \AA$; iv) modèle déduit de l'expression (4) $(+++++)$ avec $\sqrt{\left\langle z^{2}\right\rangle}=21,2 \AA$. Pour ces diverses courbes théoriques l'indice est donné par $\delta=13,3 \times 10^{-6}, \beta=1,14 \times 10^{-6}$. Note: la légère différence qui apparaît sur la valeur de $\sqrt{\left\langle z^{2}\right\rangle}$ du modèle (5) a été rendu nécessaire pour faire coïncider les valeurs d'intensité avec celles données par le modèle C. P. S. et (4) pour les angles d'incidence élevés, et semble imputable à des incertitudes de calcul.

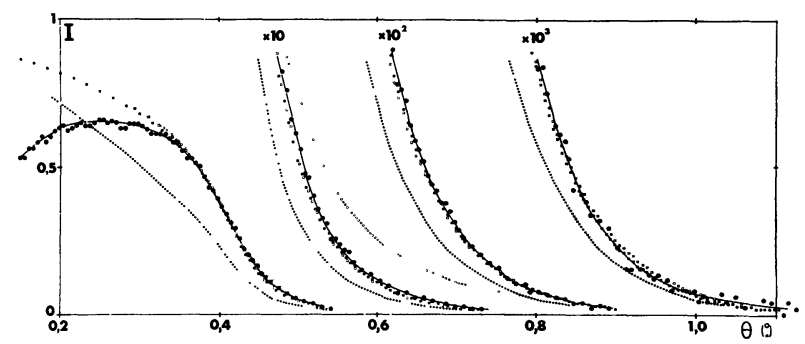

Fig. $3 b$. - Même légende que précédemment en ( $3 a)$ mais le rayonnement utilisé est celui du chrome $(\lambda=2,2896 \AA)$ l'indice considéré pour le modèle plan parfait est tel que : $\delta=25,8 \times 10^{-6}, \beta=4,6 \times 10^{-6}$. Pour les autres modèles $\delta=26,8 \times 10^{-6}, \sqrt{\left\langle z^{2}\right\rangle}=20,33$ dans le modèle déduit de (5).

siques correspondantes sont de $13,1 \times 10^{-6}$ et $29,0 \times 10^{-6}$, lesquelles, après correction de dispersion anomale, deviennent : $13,25 \times 10^{-6}$ et $26,78 \times 10^{-6}$ (ces derniers indices ont été calculés à partir de la valeur moyenne des corrections $\Delta f^{\prime}$ sensiblement équivalentes publiées en $[1,2,3])$. Nous supposerons alors ces dioptres homogènes, mais rugueux, en précisant cependant que, dans tout ce qui suit, nous nous limiterons au cas d'un seul dioptre.

Nous pouvons remarquer que le rôle des rugosités ou irrégularités de surface sur la réflexion des rayons $\mathbf{X}$ peut être analysé plus aisément dans le cas des incidences assez fortes, c'est-à-dire dans un domaine angulaire tel que les coefficients de réflexion soient assez faibles pour que l'on puisse considérer que le rayonnement pénètre dans la matière sans réfraction et sans changement d'amplitude, mais où cependant les différents processus de diffusion envisagés précédemment ont encore une contribution négligeable. Les différents modèles de rugosités que l'on présentera plus loin permettent de déterminer la variance du profil rugueux et donnent, précisément dans ce domaine des angles $\theta$ élevés, des résultats comparables. Pour traiter le cas général, il serait nécessaire de connaître le spectre du profil rugueux, c'est-à-dire les variations de

$$
T_{(h)}=\frac{1}{S}\left|\iint_{S} z \exp \left[-2 \pi i\left(h_{x} \cdot x+h_{y} \cdot y\right)\right] \mathrm{d} S\right|^{2}
$$

$S$ représente ici la surface de la fente d'analyse et $h$ désigne le module du vecteur d'onde $\mathbf{h}$ associé à la rugosité.

Cependant certaines approximations peuvent rendre les calculs plus simples, en particulier si, dans le spectre, la contribution principale est assurée soit par les basses fréquences, soit par les hautes fréquences. Avant d'examiner ces deux cas particuliers nous devons définir plus précisément ce que nous entendons par basses ou hautes fréquences spatiales.

Soit $\mathbf{k}_{\mathbf{i}}$ le vecteur d'onde du rayonnement incident et $\theta_{i}$ l'angle d'attaque sur la surface. Une rugosité de vecteur $\mathbf{h}$ donnera naissance à deux ondes diffractées de vecteurs d'onde $\mathbf{k}_{\mathrm{d}}$, de même module que $\mathbf{k}_{\mathbf{i}}$ (soit $\left|\mathbf{k}_{\mathbf{i}}\right|=\left|\mathbf{k}_{\mathrm{d}}\right|=k_{0}$ ) et de composantes tangentielles dans le plan $x O y$ telles que $\left(\mathbf{k}_{\mathrm{d}}\right)_{\mathrm{t}}=\left(\mathbf{k}_{\mathrm{i}}\right)_{\mathrm{t}} \pm \mathbf{h}$. (Voir schéma.)

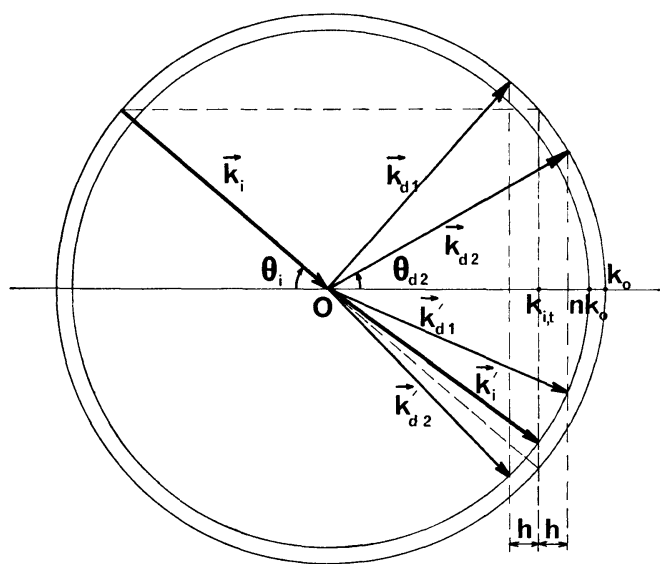

ScHÉmA 3.3. - Construction des rayons diffractés par une rugosité de vecteur d'onde associé $h$.

Si les angles $\theta_{\mathrm{d}}$ relatifs à ces ondes diffractées sont tels que la variation relative $\left(\theta_{\mathbf{d}}-\theta_{\mathbf{i}} / \theta_{\mathbf{i}}\right)$ soit petite devant 1 , nous dirons que l'ondulation correspondante est de grande période spatiale (ou de basse fréquence), et inversement, si la variation est grande devant 1 , l'ondulation sera de courte période (haute fréquence spatiale).

Remarquons que nous pouvons substituer à ce critère la condition : $h /(1-n) k_{0}$ (ou encore $h /\left(k_{0} \delta\right)$ ) petit ou grand devant 1 , ce qui peut se voir de la manière suivante. 
En observant d'abord que la zone angulaire qui nous intéresse pour la simulation des courbes expérimentales est celle où les coefficients de réflexion sont importants (nous savons que dans la zone où ces coefficients deviennent très faibles, ces différents modèles conduisent à des résultats équivalents), on en déduit que le rapport des composantes normales des vecteurs d'onde incident $k_{\mathbf{i} z}$ et réfracté $k_{\mathbf{i} z}^{\prime}$ doit être suffisamment différent de 1 (on rappelle que le coefficient de réflexion s'exprime par

$$
\left(k_{\mathrm{i} z}-k_{\mathbf{i} z}^{\prime} /\left(\left(k_{\mathrm{iz}}+k_{\mathrm{i} z}^{\prime}\right)\right)\right. \text {. }
$$

Or la construction géométrique du rayon réfracté permet d'écrire les relations :

$$
k_{\mathrm{i} z}^{2}=\left(k_{0}-k_{\mathrm{it}}\right)\left(k_{0}+k_{\mathrm{it}}\right)
$$

et

$$
k_{\mathrm{i} z}^{\prime 2}=\left(n k_{0}-k_{\mathrm{it}}\right)\left(n k_{0}+k_{\mathrm{it}}\right)
$$

et par suite

$$
\frac{k_{\mathrm{i} z}}{k_{\mathrm{i} z}^{\prime}}=\sqrt{\frac{k_{0}-k_{\mathrm{it}}}{n k_{0}-k_{\mathrm{it}}}}=\frac{1}{\sqrt{1-\frac{(1-n) k_{0}}{k_{0}-k_{\mathrm{it}}}}} .
$$

Il faudra donc que : $\left(k_{0}-k_{\mathrm{it}}\right)$ soit de l'ordre de grandeur de $(1-n) k_{0}$ (ou de $k_{0} \delta$ ). La construction géométrique des rayons diffractés montre que $\theta_{\mathrm{d}}$ et $\theta_{\mathbf{i}}$ seront très voisins si la condition $h \ll\left(k_{0}-k_{\mathrm{it}}\right)$ est vérifiée, et très différents dans le cas contraire. Par conséquent, les basses fréquences pourront être définies par l'inégalité $h \ll k_{0} \delta$ et les hautes fréquences par $h \gg k_{0} \delta$.

Pour un substrat de float-glass attaqué par le rayonnement $\mathrm{K}_{\alpha_{1}}$ du cuivre, $k_{0} \delta$ est égal à $0,05 \mu \mathrm{m}^{-1}$. Dans le cas de l'or on aurait $k_{0} \delta=0,3 \mu \mathrm{m}^{-1}$.

Ainsi, la période spatiale de référence est-elle de l'ordre du micron, c'est-à-dire beaucoup plus élevée que la longueur d'onde du rayonnement $\mathrm{X}$ incident et guère éloignée des valeurs correspondantes pour l'optique visible où l'on ne travaille pas sous incidence rasante.

Examinons les deux cas particuliers évoqués précédemment.

3.3.1 Prédominance des basses fréquences spectrales. - Nous savons qu'en général les spectres de rugosités présentent un pic très intense pour les fréquences spatiales très basses (disons, inférieures au $\mathrm{mm}^{-1}$ ). Ce pic est dû aux ondulations macroscopiques que l'on met en évidence aisément par interférométrie en lumière visible à l'aide de miroirs plans étalons et qui correspondent aux écarts de planéité des surfaces, même les mieux polies. De telles ondulations peuvent diffracter dans la fente de mesure et il est bien évident que les fréquences spatiales correspondantes varieront avec l'angle $\theta_{2}$ de détection.

Aussi nous désignerons par $v_{\mathrm{s}\left(\theta_{2}\right)}$ la fréquence spatiale limite supérieure qui passe dans la fente.
Nous convenons d'éliminer du profil rugueux ces ondulations macroscopiques désignées généralement sous le vocable écarts de forme. La rugosité de surface apparaît ainsi comme l'ensemble des irrégularités des profils réels qui forment le relief de la surface par rapport à ces ondulations.

Nous supposons que dans le spectre des irrégularités telles que définies précédemment, la contribution des très basses fréquences spatiales (de fréquences inférieures à $\left.v_{\mathrm{s}\left(\theta_{2}\right)}\right)$ reste faible et qu'on peut admettre que tout se passe comme si la fente de mesure était d'ouverture infiniment petite pour les rugosités et ne recueillait pas la lumière diffractée.

$\mathrm{Si}$ dans le spectre le poids des basses fréquences spatiales (c'est-à-dire des fréquences supérieures à $\left.v_{\mathrm{s}\left(\theta_{2}\right)}\right)$ est prépondérant, la diffraction engendrée par les rugosités s'effectue dans une direction proche de celle de la réflexion spéculaire. Nous retrouvons alors le problème traité par $P$. Croce, $L$. Névot et B. Pardo [9]. Aussi ne ferons-nous que rappeler qu'on peut admettre qu'en chaque point de la surface du dioptre, les amplitudes des ondes sont celles que donneraient localement les équations de Fresnel sur le plan idéal de même cote $z$ que le point considéré (ce qui revient à négliger les variations de pente du profil rugueux) à condition toutefois de tenir compte du déphasage afférent à la cote $z$ (voir schéma). Si on

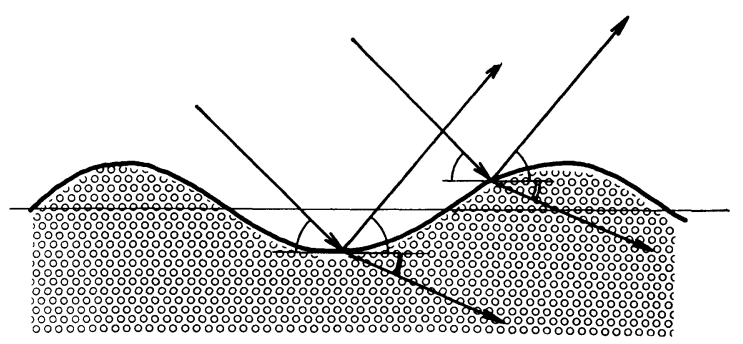

Schéma 3.3.1. - Construction des rayons respectivement réfléchis et réfractés, lorsque le spectre des rugosités révèle une prédominance des basses fréquences spatiales.

adopte, en outre, une loi de distribution gaussienne pour les cotes des rugosités (relativement au plan moyen du profil rugueux) on montre facilement que l'intensité réfléchie spéculairement par le dioptre rugueux est, par rapport à celle que l'on aurait pour un dioptre parfaitement plan, atténuée par le facteur exponentiel bien connu :

$$
\exp \left(-\frac{16 \pi^{2}}{\lambda^{2}}<z^{2}>\sin ^{2} \theta\right)
$$

Dans le cas de la réflexion diffuse, ce facteur s'écrit :

$$
\operatorname{sxp}\left[-\frac{4 \pi^{2}}{\lambda^{2}}<z^{2}>\left(\sin \theta_{1}+\sin \theta_{2}\right)^{2}\right] \text {. }
$$

3.3.2 Prédominance des hautes fréquences spatiales. - L'effet de perturbation du champ électrique par les ondes diffractées, au voisinage du dioptre, peut 
être négligé. En effet, on peut montrer que l'une des ondes diffractées est évanescente et que sa contribution, dans la direction du faisceau réfléchi, est en quadrature avec l'onde principale; quant à l'autre, elle fait avec la surface un angle suffisamment élevé (puisque $\theta_{\mathrm{d}} \gg \theta_{\mathrm{i}}$ ) et se trouve, par suite, peu excitée au niveau de la surface. Par conséquent on ne conservera que les ondes principales, à savoir, dans le vide (ou l'air) l'onde incidente (de vecteur $\mathbf{k}_{\mathrm{i}}$ ) et l'onde réfléchie, et dans la matière, l'onde réfractée (de vecteur $\mathbf{k}^{\prime}$ ), ces ondes étant déterminées par les équations de Fresnel au niveau du plan moyen du profil rugueux (voir schéma).

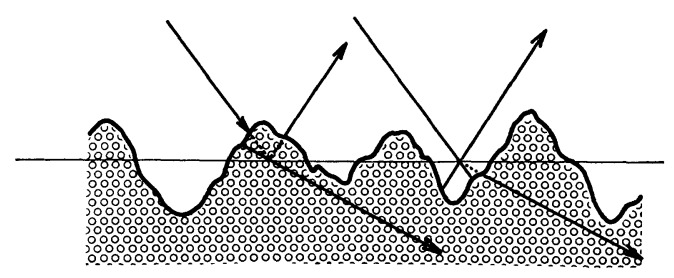

SCHÉmA 3.3.2. - Construction des rayons respectivement réfléchis et réfractés, lorsque le spectre des rugosités révèle une prédominance des hautes fréquences spatiales.

Dans le cas de la réflexion spéculaire, on montre alors que l'intensité réfléchie relative au modèle plan parfait doit être multipliée par l'expression :

$$
\begin{aligned}
\mid 1+\exp \left[-2 \pi^{2}<z^{2}>\left(k_{\mathrm{i}, z}^{\prime}+k_{\mathrm{i}, z}\right)^{2}\right]- \\
-\left.\exp \left[-2 \pi^{2}<z^{2}>\left(k_{\mathrm{i}, z}^{\prime}-k_{\mathrm{i}, z}\right)^{2}\right]\right|^{2} .
\end{aligned}
$$

Dans le cas de la réflexion diffuse, l'expression de ce facteur peut être approximée par :

$$
\begin{aligned}
& \frac{1}{4} \mid \frac{1}{1+r_{\mathrm{d}}} \exp \left[-2 \pi^{2}<z^{2}>\left(k_{\mathrm{i}, z}^{\prime}+k_{\mathrm{d}, z}\right)^{2}\right]+ \\
& \quad+\frac{r_{\mathrm{d}}}{1+r_{\mathrm{d}}} \exp \left[-2 \pi^{2}<z^{2}>\left(k_{\mathrm{i}, z}^{\prime}-k_{\mathrm{d}, z}\right)^{2}\right] \\
& \quad+\frac{1}{1+r_{\mathrm{i}}} \exp \left[-2 \pi^{2}<z^{2}>\left(k_{\mathrm{d}, z}^{\prime}+k_{\mathrm{i}, z}\right)^{2}\right] \\
& \quad+\left.\frac{r_{\mathrm{i}}}{1+r_{\mathrm{i}}} \exp \left[-2 \pi^{2}<z^{2}>\left(k_{\mathrm{d}, z}^{\prime}-k_{\mathrm{i}, z}\right)^{2}\right]\right|^{2}
\end{aligned}
$$

où $r_{\mathrm{i}}$ et $r_{\mathrm{d}}$ désignent les facteurs de réflexion en amplitude [tels que $\left(1+r_{\mathrm{i}}\right)$ et $\left(1+r_{\mathrm{d}}\right)$ soient égaux respectivement aux facteurs de transmission $\tau_{\mathrm{i}}$ et $\tau_{\mathrm{d}}$ ] pour des angles d'incidences $\theta_{\mathrm{i}}$ et $\theta_{\mathrm{d}} \cdot k_{\mathrm{d}, z}$ et $k_{\mathrm{d}, z}^{\prime}$ représentent les composantes normales des vecteurs d'onde de l'onde diffractée que l'on aurait dans le vide et dans la matière pour l'incidence $\theta_{\mathbf{d}}$. Nous n'essaierons pas ici de donner une justification de ces deux dernières formules qui s'appuient sur la méthode exposée par P. Croce, L. Névot, B. Pardo [6]. Comme nous le verrons, l'accord est assez bon avec l'expérience et cela dans un domaine angulaire élevé où les ondes dans la matière sont parfois évanescentes, parfois progressives (les valeurs de $r, k$, sont évidemment complexes et tiennent également compte de l'absorption). Par la méthode des réseaux, on peut confronter les formules $\left(4^{\prime}\right)$ et $\left(5^{\prime}\right)$ avec l'expérience car la région du spectre des rugosités ainsi étudiée est fixe, ce qui introduit un facteur constant pour toute la courbe. Remarquons que, comme il se doit, ces formules ne changent pas quand on échange l'angle d'incidence et l'angle de diffusion et que les deux modèles $\left(4^{\prime}\right)$ et $\left(5^{\prime}\right)$ tendent l'un vers l'autre pour les angles élevés, les valeurs de $r_{\mathrm{i}}, r_{\mathrm{d}}, k_{\mathrm{i} z}^{\prime}-k_{\mathrm{i} z}$, etc... devenant alors très faibles. La même méthode nous permet de trouver d'autres formules approchées que nous n'avons pas encore essayées.

Précisons également que le fait de travailler avec des fentes à la place de trous n'apporte aucun changement essentiel à ce qui vient d'être présenté.

Le spectre des rugosités est à deux dimensions, mais les vecteurs d'onde associés aux rugosités qui vont diffracter, dans la fente réceptrice, le rayonnement issu d'un point de la fente source, diffèrent de certains vecteurs, lesquels changent extrêmement peu lorsque $\theta_{\mathrm{i}}$ et $\theta_{\mathrm{d}}$ varient en étant astreints à vérifier la loi $\cos \theta_{\mathbf{i}}-\cos \theta_{\mathrm{d}}=$ Cte, et en restant petits. Les composantes normales des vecteurs d'onde des diverses ondes incidentes, provenant de la fente source, ou diffractées, passant dans la fente réceptrice, seront voisines les unes des autres. On retrouvera donc sensiblement les mêmes facteurs $(4),\left(4^{\prime}\right),(5)$ et $\left(5^{\prime}\right)$.

On peut constater aussi que l'expression (5), appliquée à des rugosités de faible amplitude, conduit à des résultats comparables à ceux que l'on obtiendrait à l'aide du modèle de couche de passage stratifiée (C. P. S.) où l'indice varie avec la cote $z$ suivant la fonction d'erreur, modèle que nous avons proposé antérieurement (P. Croce et L. Névot $[5,10])$. Rappelons que la relation d'équivalence entre l'épaisseur $L$ de cette couche de passage et la valeur quadratique moyenne $\sqrt{\left\langle z^{2}\right\rangle}$ des rugosités, est :

$$
L=4,52 \sqrt{\left\langle z^{2}\right\rangle} \text {. }
$$

4. Résultats expérimentaux. - 4.1 EXAMEN DE LA RÉFLEXION SPÉCULAIRE. - Nous avons rassemblé dans le tableau II les valeurs d'indice et celles des paramètres qui caractérisent les rugosités (c'est-à-dire, $L$, la hauteur moyenne créte à crête des rugosités dans le modèle C. P. S., ou $\sqrt{\left\langle z^{2}\right\rangle}$ tel que déduit de l'expression (5)) obtenues à la suite des simulations des courbes expérimentales de réflexion spéculaire fournies par des échantillons massifs de titane (Fig. 3a et $3 b$ ), de silicium (Fig. 4) et de germanium (Fig. 5 et 6), attaqués par le rayonnement $K_{\alpha_{1}}$ du cuivre ou celui du chrome. De ces diverses simulations se dégagent les remarques suivantes :

i) Equivalence des différents modèles proposés, pour les angles d'incidence élevés (à $L$ ou $\sqrt{\left\langle z^{2}\right\rangle}$ constants et avec les mêmes indices évidemment). 


\section{TABLEAU II}

\begin{tabular}{|c|c|c|c|c|c|c|c|c|}
\hline Substance & $\lambda \AA$ & $\begin{array}{c}10^{6} \delta \\
\text { modèle plan } \\
\text { parfait }\end{array}$ & $\begin{array}{l}L \AA \\
\text { modèle } \\
\text { C. P. S. } \\
-\end{array}$ & $\begin{array}{c}\sqrt{<z^{2}>} \\
\text { modèle } \\
(5) \\
-\end{array}$ & $\begin{array}{l}10^{6} \delta^{*} \\
\text { exp. }\end{array}$ & $\begin{array}{c}10^{6} \delta \\
\text { th. } \\
\text { classique } \\
-\end{array}$ & $\underline{\Delta f^{\prime}}$ & $\begin{array}{c}10^{6} \delta^{*} \\
\text { th. }\end{array}$ \\
\hline Titane & $\begin{array}{l}1,5405 \\
2,2896\end{array}$ & $\begin{array}{l}12,8 \pm 0,2 \\
25,8 \pm 0,4\end{array}$ & $\begin{array}{l}96 \\
92\end{array}$ & $\begin{array}{l}21,9 \\
20,33\end{array}$ & $\begin{array}{l}13,30 \pm 0,15 \\
26,8 \pm 0,4\end{array}$ & $\begin{array}{l}13,12 \\
28,99\end{array}$ & $\begin{array}{l}+0,21[1,2,3] \\
-1,68[1,2,3]\end{array}$ & $\begin{array}{l}13,25 \\
26,78\end{array}$ \\
\hline Silicium & 1,5405 & $7,6 \pm 0,1$ & 47 & 10,6 & $7,6 \pm 0,1$ & 7,44 & $\begin{array}{l}+0,279[11] \\
+0,244[3]\end{array}$ & $\begin{array}{l}7,59 \\
7,57\end{array}$ \\
\hline Germanium & 1,5405 & $\begin{array}{l}14,0 \pm 0,15 \\
14,05 \pm 0,15\end{array}$ & $\begin{array}{r}116 \\
94\end{array}$ & $\begin{array}{l}26,8 \\
21,5\end{array}$ & $\begin{array}{l}14,35 \pm 0,15 \\
14,35 \pm 0,15\end{array}$ & 15,05 & $-1,26[1,2,3]$ & 14,46 \\
\hline
\end{tabular}

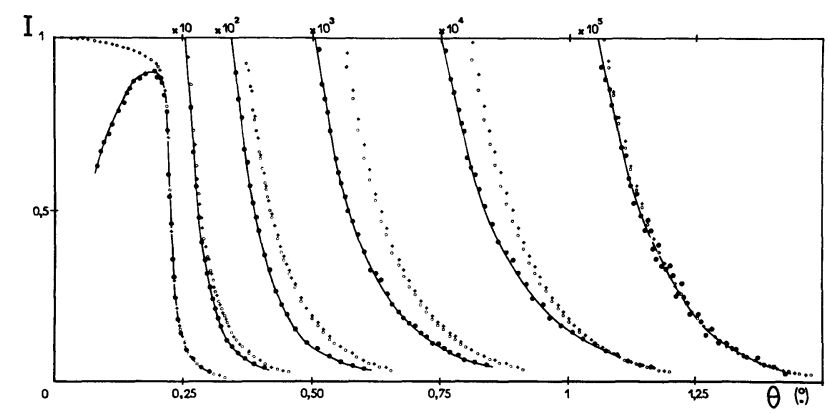

Fig. 4. - Courbe de réflexion spéculaire ( $\bullet-\bullet \bullet)$ obtenue avec le rayonnement $\mathrm{CuK}_{\alpha_{1}}$, sur un échantillon massif de silicium. Les courbes théoriques correspondent à $\delta=7,6 \times 10^{-6}$, $\beta=1,7 \times 10^{-7}$, et $L=47 \AA$ (soit $\sqrt{\left\langle z^{2}\right\rangle}=10,4 \AA$ ) pour le modèle C. P. S. (o०००oo), ou $\sqrt{\left\langle z^{2}\right\rangle}=10,6 \AA$ pour le modèle déduit de $(5)(+++++)$.

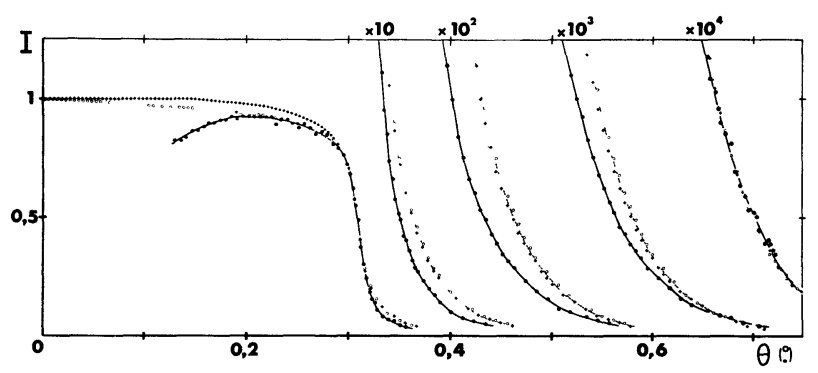

Fig. 5. - Courbe de réflexion spéculaire ( $-\bullet \bullet-\longrightarrow)$ fournie par un échantillon massif de germanium, attaqué par le rayonnement $\mathrm{CuK}_{\alpha_{1}}$. Les courbes théoriques correspondent à $\delta=14,35 \times 10^{-6}, \beta=5 \times 10^{-7}$ et $L=94 \AA$

(soit $\sqrt{\left\langle z^{2}\right\rangle}=20,77 \AA$ ) pour le modèle C. P. S. (ooooo), ou $\sqrt{\left\langle z^{2}\right\rangle}=21,51 \AA$ pour le modèle $(5)(+++++)$.

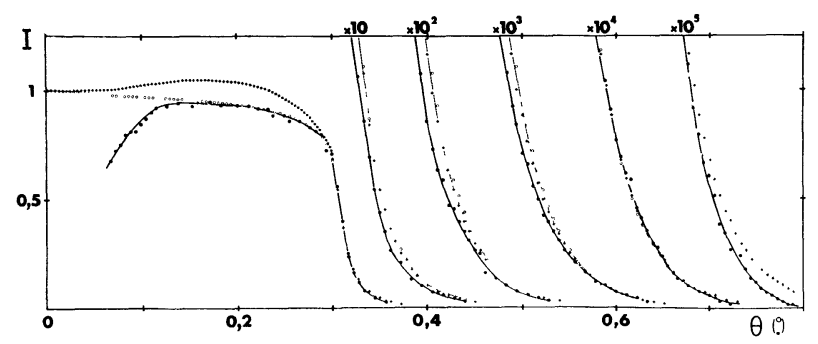

Fig. 6. - Même légende que pour la figure 5, mais pour les courbes théoriques $L=116 \AA$ (soit $\sqrt{\left\langle z^{2}\right\rangle}=25,6 \AA$ ) pour le modèle C. P. S. (o०ooo) ou $\sqrt{\left\langle z^{2}\right\rangle}=26,8 \AA$ pour le modèle $(5)(+++++)$. ii) Equivalence du modèle (5) et de C. P. S. quelque soit l'angle d'incidence, si les rugosités sont de faible amplitude (voir Fig. 4). Par contre, lorsque les rugosités sont de forte amplitude, la forme de courbe obtenue dans la zone de réflexion totale s'écarte de plus en plus, lorsque $\left\langle z^{2}\right\rangle$ croît, de celle donnée par C. P. S., cette dernière étant celle qui s'apparente le mieux à la courbe expérimentale (comparer les Fig. 5 et 6).

iii) Insuffisance du modèle (4), surtout lorsque $\theta_{\mathrm{i}}$ est voisin de $\theta_{\mathrm{c}}$.

Ceci est particulièrement frappant lorsque l'échantillon analysé est assez absorbant pour le rayonnement $\mathrm{X}$ utilisé et présente de fortes rugosités (voir Fig. $3 a$ et $3 b$ ). Par contre, comme nous l'avons constaté antérieurement [5] sur des échantillons de float-glass, bruts de fabrication, le modèle (4) est pratiquement équivalent à C. P. S. dans le cas contraire : faible absorption, faibles rugosités. (Rappelons que pour ce matériau, $L$ est compris entre 20 et $30 \AA$, soit $\sqrt{\left\langle z^{2}\right\rangle}$ de l'ordre de $5,5 \AA$, et que $\beta$ est égal à $1,3 \times 10^{-7}$ pour le rayonnement $\mathrm{K}_{\alpha_{1}}$ du cuivre.)

iv) Excellent accord des indices trouvés, lorsqu'on tient compte des rugosités (valeurs apparaissant dans la colonne $" 10^{6} \delta_{\exp }^{*} »$ du Tableau II) avec les indices théoriques obtenus après correction de dispersion anomale (colonne « $\left.10^{6} \delta_{\text {théorique }}^{*}\right)$. On remarquera, en outre, que le fait de faire intervenir la rugosité permet de rattraper l'écart d'indice, donc de densité, que l'on serait tenté de déduire pour les deux échantillons de germanium étudiés, en se basant uniquement sur les valeurs données par le modèle plan parfait.

On notera aussi que les valeurs de $L$ ou $\sqrt{\left\langle z^{2}\right\rangle}$ restent sensiblement les mêmes, que l'analyse soit faite avec le rayonnement $k_{\alpha_{1}}$ du cuivre ou avec celui du chrome (Fig. $3 a$ et $3 b$ ).

v) On observera que les diverses courbes expérimentales traduisent une chute imposante de l'intensité réfléchie lorsque l'angle d'incidence tend vers 0 , et ceci sur une zone angulaire plus ou moins étendue, alors que cette intensité devrait tendre vers la valeur mesurée dans le faisceau direct (donc vers 1 , en valeur relative). Une telle anomalie résulte d'effets géométriques : d'une part, plus la surface éclairée est importante, plus les écarts de planéité du spécimen risquent 
de prendre une part importante dans la perturbation de la réflexion du faisceau incident; d'autre part, même un échantillon idéalement plan n'interceptera complètement le faisceau incident (et par conséquent, ne pourra le réfléchir totalement) que pour des angles d'incidence supérieurs à une certaine valeur $\theta_{\mathrm{m}}$, du fait de sa dimension latérale finie. Pour les échantillons que nous avons analysés $\theta_{\mathrm{m}}$ est égal à $0,26^{\circ}$ dans le cas du titane (surface circulaire de $2 \mathrm{~cm}$ de diamètre), $0,13^{\circ}$ pour le silicium (surface circulaire de $3 \mathrm{~cm}$ de diamètre) et $0,11^{\circ}$ pour le germanium (surface sensiblement carrée, de $3 \mathrm{~cm}$ de côté, pour les deux spécimens).

4.2 EXAMEN DE LA RÉFLEXION DIFFUSE (LOI DES RÉSFAUX). - Nous présentons sur les figures $7(a, b, c$, d) les courbes de réflexion diffuse (avec la loi des réseaux) obtenues avec le rayonnement $\mathrm{CuK}_{\alpha_{1}}$, sur l'un des échantillons de germanium (précédemment étudié en réflexion spéculaire et pour lequel nous avons trouvé $\sqrt{\left\langle z^{2}\right\rangle}=21,5 \AA$ ) pour quelques fréquences spatiales particulières. Dans le cas du germanium et avec le rayonnement $\mathrm{CuK}_{\alpha_{1}}, k_{0} \delta$ prend la valeur $0,093 \mu \mathrm{m}^{-1}$. Les fréquences étudiées pourront être considérées respectivement comme basse (Fig. 7a, $v=0,037 \mu^{-1}$ ), moyennes (Fig. $7 b, v=0,097 \mu \mathrm{m}^{-1}$ et Fig. $7 c, v=0,183 \mu \mathrm{m}^{-1}$ ) et haute (Fig. $7 d$, $\left.v=0,427 \mu \mathrm{m}^{-1}\right)$. Nous avons essayé de simuler les courbes expérimentales à l'aide des expressions théoriques $\left(4^{\prime}\right)$ et $\left(5^{\prime}\right)$ en conservant les valeurs d'indice et de rugosité fournies par l'analyse de la courbe de réflexion spéculaire et en tenant compte du facteur de géométrie (on ne doit considérer en effet, que la surface éclairée utile, c'est-à-dire qui diffuse effectivement dans le compteur; puisque les ouvertures des fentes source et analyse sont identiques $(60 \mu \mathrm{m})$ et que, pour les courbes présentées, $\theta_{2}$ reste supérieur à $\theta_{1}$, la surface éclairée varie comme $1 / \sin \theta_{2}$ ).

Notons aussi que les courbes théoriques ont été ajustées afin de faire coïncider les valeurs maximales d'intensité avec celles données par l'expérience. En ce qui concerne le modèle $\left(4^{\prime}\right)$ la forme du pic de diffusion est assez bien reproduite pour les très basses fréquences (Fig. 7a), mais le pic est déplacé vers les incidences faibles pour les fréquences élevées. On constate aussi que ce modèle prédit, de façon systématique (c'est-à-dire quelle que soit la fréquence considérée), une décroissance trop lente de l'intensité diffusée pour les incidences élevées.

La simulation obtenue à l'aide du modèle $\left(5^{\prime}\right)$ est excellente dans le domaine des hautes fréquences. Par contre pour les basses fréquences, le pic de diffusion est fortement décalé vers les incidences plus faibles.

Le pic de diffusion de la figure $7 a$ révèle l'existence de deux maximums et chacun d'eux correspond sensiblement au moment où la valeur de l'angle, soit d'incidence, $\theta_{1}$, soit de diffusion, $\theta_{2}$, équivaut à $\theta_{c}$. On a bien là la confirmation du principe de réciprocité. Par suite, il apparaît comme nécessaire que les

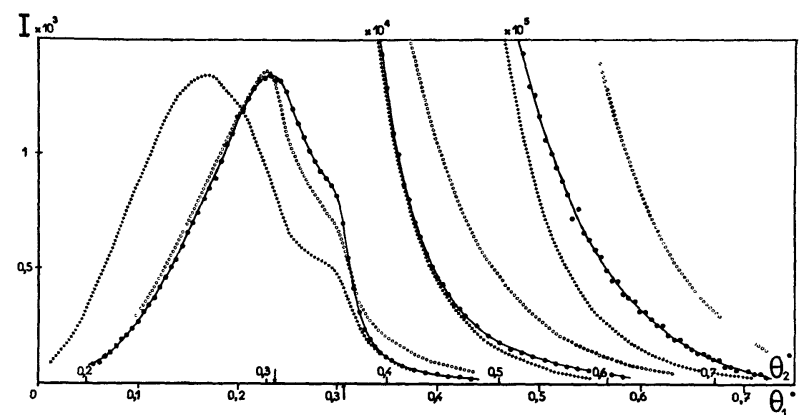

Fig. 7a. - Courbe de réflexion diffuse (en loi des réseaux) obtenue avec le rayonnement $\mathrm{CuK}_{\alpha_{1}}$, sur l'échantillon de germanium dont la courbe de réflexion spéculaire est présentée sur la figure 5. Les courbes théoriques correspondent au modèle $\left(4^{\prime}\right)$ avec $\sqrt{\left\langle z^{2}\right\rangle}=20,77 \AA$ (00000), et au modèle $\left(5^{\prime}\right)$ avec $\sqrt{\left\langle z^{2}\right\rangle}=21,51 \AA(+++++)$. La fréquence spatiale est de $0,037 \mu \mathrm{m}^{-1}$. Note : Les deux flèches sur l'axe des abscisses indiquent les positions pour lesquelles les valeurs de $\theta_{1}$ (angle d'incidence) et de $\theta_{2}$ (angle de détection) correspondent précisément à $\theta_{\mathrm{c}}\left(\theta_{\mathrm{c}}=\sqrt{2 \delta}=5,36 \times 10^{-3} \mathrm{rd}\right)$.

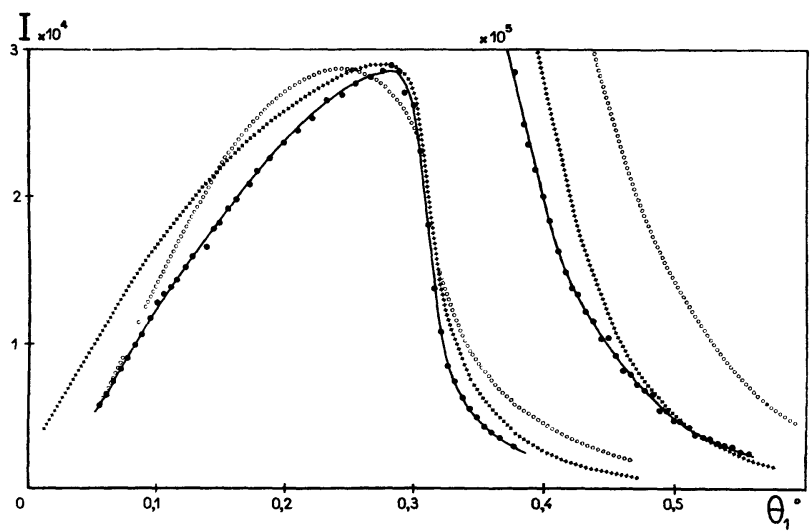

Fig. 7b. - Même légende que précédemment en $7 a$, la fréquence spatiale étant égale à $0,097 \mu \mathrm{m}^{-1}$.

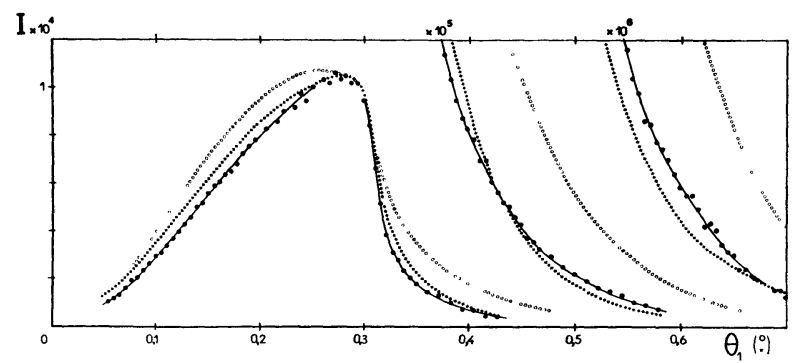

Fig. 7c. - Même légende qu'en $7 a$, avec pour fréquence spatiale $0,183 \mu \mathrm{m}^{-1}$.

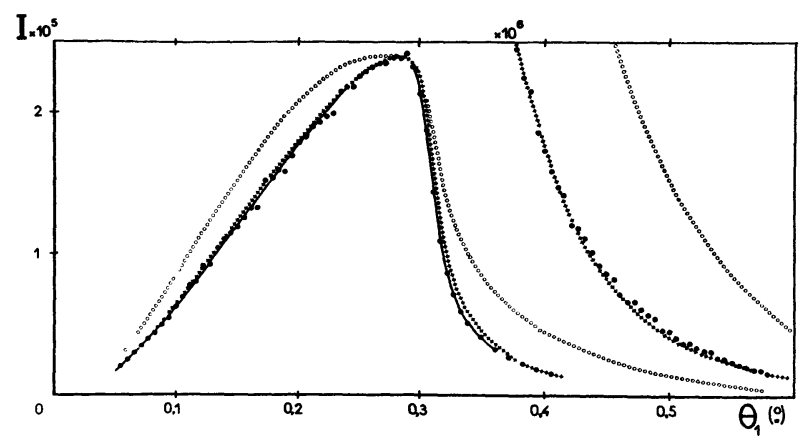

FIG. $7 d$. - Même légende qu'en $7 a$, avec pour fréquence spatiale $0,427 \mu \mathrm{m}^{-1}$. 
expressions théoriques, même approchées, que l'on pourrait établir, vérifient ce principe.

En examinant les valeurs respectives des angles $\theta_{1}$ et $\theta_{2}$ de la figure $7 a$, on constate qu'il sera difficile d'étudier la diffusion par les fréquences spatiales bien plus basses que $0,037 \mu \mathrm{m}^{-1}$. En effet, ces angles deviennent assez rapidement trop voisins et la fente de mesure recueille alors une partie du faisceau réfléchi spéculairement.

D'un autre côté, l'intensité diffusée obtenue avec la fréquence de $0,427 \mu \mathrm{m}^{-1}$ apparaît déjà comme très faible. Les effets de perturbation, dus aux divers processus de diffusion évoqués en 3.1 et 3.2 , risquent donc de compliquer quelque peu l'interprétation, déjà délicate, des courbes de diffusion relatives aux fréquences spatiales très élevées.

Le domaine de fréquences spatiales que l'on peut explorer semble ainsi relativement étroit. Aurait-on avantage à changer de longueur d'onde pour le rayonnement incident?

Nous savons que si la loi de dispersion classique (c'est-à-dire, celle qui correspond à l'électron libre) était valable, il existerait une loi de similitude entre $\lambda$ et $\theta$ [12]. Ainsi les courbes de réflexion spéculaires relatives à deux rayonnements de longueurs d'onde $\lambda_{1}$ et $\lambda_{2}$, seraient affines l'une de l'autre avec comme rapport $\theta_{2} / \theta_{1}=\lambda_{2} / \lambda_{1}$. Donc en des points homologues les composantes normales des divers vecteurs d'onde sont les mêmes et la difficulté pour traiter, dans le cas général, du rôle des rugosités, reste inchangée $\left({ }^{2}\right)$. Cependant on peut remarquer que $k_{0} \delta$ varie comme $\lambda$ (puisque $\delta$ est proportionnel à $\lambda^{2}$, si on néglige les termes de dispersion anomale). De ce fait, lorsque $\lambda$ augmente, le spectre des rugosités a tendance à devenir de basses fréquences. Par ailleurs nous savons que le coefficient d'absorption varie comme $\lambda^{m}$ (avec $m$ compris entre 2 et 3 ). La profondeur utile 3 pour des incidences homologues est donc plus faible, lorsque $\lambda$ augmente. On améliore ainsi les conditions d'étude des irrégularités de surface. Rappelons cependant que l'absorption par l'air entraîne, malheureusement, une perte de flux appréciable pour ces longueurs d'onde plus élevées (la comparaison des intensités des courbes des figures $3 a$ et $3 b$ est assez éloquente à cet égard) et qu'il devient indispensable d'effectuer de telles analyses sous vide.

5. Conclusion. - Il est assez généralement admis que les mesures d'épaisseur par analyse de la réflexion spéculaire rasante de rayons $\mathrm{X}$ sont les plus précises qu'il soit possible d'effectuer dans le domaine d'épaisseur allant de quelques dizaines à quelques centaines d'angströms. Cette méthode a aussi l'avantage d'être non destructive mais par contre elle requiert des sur-

(2) Par contre, les contraintes imposées par les écarts de forme seraient moindres, puisqu'on pourrait alors utiliser une ouverture de fente plus grande. faces suffisamment planes et elle ne permet pas la présence de fluide dense sur la surface libre empêchant ainsi des études en milieu liquide. On peut l'utiliser aussi pour déterminer les indices, et par suite, les densités, des matériaux étudiés, à condition toutefois de tenir compte des rugosités ou irrégularités de surface. A cet effet, nous avons proposé quelques modèles de rugosités et précisé leur domaine de validité. On améliore ainsi considérablement la simulation des courbes expérimentales de réflexion spéculaire dans la zone des incidences de l'ordre de $\theta_{c}$ ou au contraire très élevées, mais on notera toutefois qu'il peut subsister de légers écarts dans la zone intermédiaire. Dans de nombreux cas, il est possible d'attribuer ces écarts à l'existence de couches superficielles assez épaisses d'oxyde ou, de façon plus générale, de contamination, laquelle peut se produire lors de la remise à l'air libre pour les dépôts réalisés sous vide, ou résulter des procédés de polissage de surfaces des échantillons massifs, et être mise en évidence, par exemple, par des expériences d'électrons Auger, ou par microanalyse nucléaire [13]. Ainsi, cette dernière technique, appliquée à des échantillons de titane poli à l'alumine $(0,05 \mu \mathrm{m})$, sous eau, et sur poix de Bourgogne (méthode de polissage que nous avons utilisée pour le titane et le germanium), révèle que la contamination reste faible en oxygène, et on constate bien sur les courbes des figures $3 a$ et $3 b$ que la simulation reste excellente dans la zone des incidences moyennes $\left(\theta \gtrsim \theta_{\mathrm{c}}\right)$.

Pour des échantillons de silicium (Fig. 4) et de germanium (Fig. 6) nous aurions pu essayer de bâtir un modèle de couche superficielle, en nous basant sur le fait qu'aucune frange, ou début de frange, n'apparaissant sur la courbe de réflexion spéculaire, une telle couche n'est certes pas homogène, mais peut être, malgré tout, stratifiée.

C'est ce que nous avons entrepris dans le cas de substrats de verre poli (borosilicate crown) où le seul fait de tenir compte des rugosités ( $L$ est de l'ordre de $40 \AA$ ) se révèle nettement insuffisant dans la zone des incidences moyennes. La variation d'indice que l'on est amené ainsi à considérer reste très faible, puisqu'au voisinage immédiat de la surface la diminution de densité par rapport à la masse ne dépasse pas $10 \%$. Par contre l'épaisseur de cette zone superficielle peut atteindre dans certains cas quelques centaines d'angströms.

Précisons toutefois que l'indice affecté à la masse, et obtenu à l'aide des méthodes exposées précédemment, n'est pas (ou très peu) modifié lorsqu'on introduit de telles couches superficielles.

Indiquons aussi que nous avons adopté pour la simulation de ces couches, un modèle d'empilement de couches homogènes mais de densités et épaisseurs variées (méthode déjà utilisée par L. G. Parratt [8]).

Une autre remarque importante se dégage de ces simulations : on ne peut conserver l'accord avec la courbe expérimentale dans la zone des incidences 
élevées, qu'en attribuant à cette couche superficielle la même rugosité que précédemment.

Il est ainsi possible d'obtenir des informations aussi bien structurelles que géométriques à l'aide des études par réflexion rasante de rayons $X$. D'autres exemples d'application de cette technique pourront être trouvés dans la bibliographie annexe, que nous avons reconstituée à partir de l'année 1970, laquelle permettra de retrouver les références des articles plus anciens qui traitent de ce sujet.

\section{Bibliographie}

[1] Dauben, C. H., Templeton, D. H., Acta Crystallogr. 8 (1955) 841.

[2] Cromer, D. T., Acta Crystallogr. 18 (1965) 17.

[3] Cromer, D. T., Liberman, D., J. Chem. Phys. 53 (1970) 1891.

[4] Voir par exemple :

Compton, A. H., Allison, S. K., $X$-Rays in theory and experiment (D. Van Nostrand, Company, Inc, Princeton) 1935.

Born, M., Wolf, E., Principles of Optics (Pergamon Press, Oxford) 1964.

[5] Névot, L., Croce, P., J. Appl. Crystallogr. 8 (1975) 304.
[6] Croce, P., Névot, L., Pardo, B., C. R. Hebd. Séan. Acad. Sci. B 274 (1972) 803-855.

[7] Alichanow, A. T., Arizmowic, L. A., Z. Phys. 82 (1933) 489.

[8] Parratt, L. G., Phys. Rev. 95 (1954) 359.

[9] Croce, P., Névot, L., Pardo, B., Nouv. Revue Opt. Appl. 3 (1972) 37.

[10] Croce, P., Névot, L., J. Appl. Crystallogr. 7 (1974) 125.

[11] Wagenfeld, H., KühN, J., Guttman, A. J., $1^{\text {er }}$ Congrès Européen de Crystallographie Bordeaux, Sept. 1973.

[12] Croce, P., Pardo, B., Nouv. Revue Opt. Appl. 4 (1970) 229.

[13] Béranger, G., David, D., Garcia, E. A., Lucas, X., Revue Phys. Appl. 10 (1975) 87.

\section{Annexe bibliographique}

Croce, P., Devant, G., Sere, M. G. et Verhaeghe, M. F., Surf. Sci. 22 (1970) 173-186.

BLET, G., Rev. Tech. Thomson CSF 3 (1971) 110-129.

RenNer, O., Czech. J. Phys. B 22 (1972) 1007-1016.
Segmüller, A., Thin Solid Films 18 (1973) 287-294.

MalyuKov, B. A., Korolev, V. E., Meas. Tech. 6 (1973) 833835 traduit de Izmeritel' naya Teckhnika 6 (1973) 27-28. Spiller, E., Segmuller, A., Appl. Phys. Lett. 24 (1974) 60-61. 